\title{
INTRINSIC STRONG SHAPE FOR PARACOMPACTA
}

\author{
Beti Andonovic And Nikita Shekutkovski \\ Ss Cyril and Methodius University, Macedonia
}

Dedicated to Sibe Mardešić

\begin{abstract}
In this paper an intrinsic definition of strong shape for paracompact topological spaces is presented. At first a coherent proximate net $f: X \rightarrow Y$ is defined, indexed by finite subsets of normal coverings of $Y$, and then there is a homotopy between two coherent proximate nets defined. A definition of composition of classes of homotopies between two coherent proximate nets $\underline{f}: X \rightarrow Y$ and $\underline{g}: Y \rightarrow Z$ is given. Then it is proved that for any other choice of corresponding coverings, a function is obtained that is in the same class with the previously defined composition. The strong shape category is obtained, with paracompacta as objects, and the homotopy classes of coherent proximate nets as morphisms.
\end{abstract}

\section{INTRODUCTION}

The shape theory has shown to be more appropriate tool than homotopy theory when study of spaces with bad local behavior is involved ([2, 6-8, $10])$. The strong shape theory is a strengthening of shape theory $([3,6])$. The first definition of strong shape for compact metric spaces is given in [9] by embedding metric compacta in Hilbert cube. In $[6,8]$ strong shape is described for topological spaces approximating the space by inverse system of polyhedra (ANRs). Both approaches follow the corresponding approaches in shape theory. For equivalence of different approaches for metric compacta we refer to $[6]$.

2010 Mathematics Subject Classification. 55P55, 54C56, 55Q07.

Key words and phrases. Coherent proximate net, covering, homotopy class, strong shape.

The participation of authors is equal. 
The intrinsic approach to shape does not use any approximation of spaces. The intrinsic definition of strong shape for compact metric spaces is presented in [11].

The definition of strong shape in [11] is based on the notion of strong proximate sequence.

The sequence of pairs $\left(f_{n}, f_{n, n+1}\right)$ of functions $f_{n}: X \rightarrow Y$ and $f_{n, n+1}$ : $X \times I \rightarrow Y$, is a strong proximate sequence from $X$ to $Y$, if there exists a cofinal sequence of finite coverings, $\mathcal{V}_{1} \succ \mathcal{V}_{2} \succ \cdots$ of $Y$, such that for each natural number $n, f_{n}: X \rightarrow Y$, is a $\mathcal{V}_{n}$-continuous function and $f_{n, n+1}$ : $X \times I \rightarrow Y$ is a homotopy connecting $\mathcal{V}_{n}$-continuous functions $f_{n}: X \rightarrow Y$ and $f_{n, n+1}: X \times I \rightarrow Y$.

We say that $\left(f_{n}, f_{n, n+1}\right)$ is a strong proximate sequence over $\left(\mathcal{V}_{n}\right)$.

If $\left(f_{n}, f_{n, n+1}\right)$ and $\left(f_{n}^{\prime}, f_{n, n+1}^{\prime}\right)$ are strong proximate sequences from $X$ to $Y$, than there exists a cofinal sequence of finite coverings $\left(\mathcal{V}_{n}\right)$ such that $\left(f_{n}, f_{n, n+1}\right)$ and $\left(f_{n}^{\prime}, f_{n, n+1}^{\prime}\right)$ are strong proximate sequences over $\left(\mathcal{V}_{n}\right)$.

In compact metric space, the existence of cofinal sequence of coverings $\mathcal{V}_{1} \succ \mathcal{V}_{2} \succ \cdots$, allows to define strong shape theory using only homotopies of second order.

In more general case of paracompact spaces, homotopies of all orders must be considered. In [13] the construction for (strongly) paracompact spaces is described. We form all finite sets of coverings of $Y, a=\left\{\mathcal{V}_{0}, \mathcal{V}_{1}, \ldots, \mathcal{V}_{n}\right\}$, having a maximal element (i.e. a covering that refines all other coverings of that finite set, and is not refined by any other covering of that finite set). The maximal element is denoted by $\max a$.

Finite sets of coverings with a maximal element are ordered by inclusion, and this ordering is cofinite, i.e. each $a$ has only finite number of predecessors. This fact allows composition of coherent proximate nets to be defined, although such definition is technically rather complex. In this way category of strong shape is obtained for paracompact spaces. In [1] it is shown that strong shape category of metric compacta is a subcategory of the last category.

\section{Coherent proximate nets}

Let $\Delta^{n} \subseteq R^{n}, \Delta^{n}=\left\{\left(t_{1}, t_{2}, \ldots, t_{n}\right) \mid 1 \geq t_{1} \geq t_{2} \geq \ldots \geq t_{n} \geq 0\right\}$ be the non standard $n$-simplex.

It is important to note that by a function $f: X \rightarrow Y$ we do not necessarily mean continuous function.

Let $Y$ be a paracompact space. We form all finite sets of coverings of $Y$, $a=\left\{\mathcal{V}_{0}, \mathcal{V}_{1}, \ldots, \mathcal{V}_{n}\right\}$, having a maximal element (a covering that refines all other coverings of that finite set, and is not refined by any other covering of that finite set). The maximal element is denoted by max $a$. If $a \subseteq b$, then $\max a \succ \max b$.

We define an ordering " $<$ " by $a<b$ if $a \subset b$. 
Definition 2.1. A coherent proximate net $\underline{f}: X \rightarrow Y$ consists of functions

$$
\underline{f}=\left\{f_{\underline{a}} \mid \underline{a}=\left(a_{0}, a_{1}, \ldots, a_{n}\right), a_{0}<\cdots<a_{n}\right\}
$$

such that each $f_{\underline{a}}: X \times \Delta^{n} \rightarrow Y$ is st $t^{n} \max a_{0}$-continuous and is $s t^{n-1} \max a_{0}$ continuous on $\bar{X} \times \partial \Delta^{n}$, and the following coherence condition is satisfied:

$$
f_{\underline{a}}\left(x, t_{1}, t_{2}, \ldots, t_{n}\right)=\left\{\begin{array}{ll}
f_{a_{1} \ldots a_{n}}\left(x, t_{2}, \ldots, t_{n}\right), & t_{1}=1 \\
f_{a_{0} \ldots \hat{a}_{i} \ldots a_{n}}\left(x, t_{1}, \ldots, \hat{t}_{i}, \ldots, t_{n}\right), & t_{i}=t_{i+1} \\
f_{a_{0} \ldots a_{n-1}}\left(x, t_{1}, \ldots, t_{n-1}\right), & t_{n}=0
\end{array} .\right.
$$

The coherent proximate net will be shortly denoted by $f=\left(f_{a}\right)$. Next we explain the definition in special cases $n=0$ and $n=1$. If $n=0$, for each $a_{0}$, there exists $f_{a_{0}}: X \rightarrow Y$, so that $f_{a_{0}}$ is $\max a_{0^{-}}$continuous. If $n=1$, for each $a_{0}$, there exists $f_{a_{0}}: X \rightarrow Y$, so that $f_{a_{0}}$ is $\max a_{0^{-}}$continuous and for every $a_{0}, a_{1}$, there exists $f_{a_{0} a_{1}}: \Delta^{1} \times X \rightarrow Y$, such that $f_{a_{0} a_{1}}$ is $s t\left(\max a_{0}\right)$ continuous and $f_{\underline{a}}$ is $\max a_{0^{-}}$continuous on $\partial \Delta^{1} \times X$, and also

$$
f_{a_{0} a_{1}}(0, x)=f_{a_{0}}(x), f_{a_{0} a_{1}}(1, x)=f_{a_{1}}(x) .
$$

Definition 2.2. Coherent proximate nets $\underline{f}, \underline{g}: X \rightarrow Y$ are homotopic (notation: $f \approx g$ ), if there exists a coherent proximate net $\underline{H}=\left(H_{\underline{a}}\right)$, such that $H_{\underline{a}}: \bar{X} \times \bar{\Delta}^{n} \times I \rightarrow Y$ is st ${ }^{n+1} \max a_{0}$ - continuous, $H_{\underline{a}}$ is $s t^{n} \max a_{0}-$ continuous on $X \times \partial\left(\Delta^{n} \times I\right)$, and the following conditions are satisfied:

$$
\begin{aligned}
& H_{\underline{a}}(x, \underline{t}, 0)=f_{\underline{a}}(x, \underline{t}), \\
& H_{\underline{a}}(x, \underline{t}, 1)=g_{\underline{a}}(x, \underline{t}) .
\end{aligned}
$$

The relation $f \approx g$ is an equivalence relation. This is shown in [13].

\section{Composition of COHEREnt PRoximate Nets - EXISTENCE AND UNIQUENESS}

The following two Theorems are needed for the definition of composition of coherent proximate nets. Theorem 3.2 is proved in [9] for the case $k=1$.

Theorem 3.1. If $f: x \rightarrow Y$ is $W$-continuous, then id $\times f: K \times X \rightarrow K \times$ $Y$ is $K \times \mathcal{W}$-continuous, where $K$ is compact and $K \times \mathcal{W}=\{K \times W \mid W \in \mathcal{W}\}$.

Proof. $f: X \rightarrow Y$ is $W$-continuous, and therefore it follows $\forall x \in X, \exists U$ neighborhood of $x$ and $\exists W \in \mathcal{W}$, so that $f(U) \subseteq W$.

As usually $i d \times f$ is defined by $i d \times f(k, x)=(k, f(x))$. Hence, for $(k, x) \in$ $K \times X$, there exists $U, U$ being a neighborhood of $x$, and there exists $K \times W \in$ $K \times \mathcal{W}$, so that $i d \times f(K \times U) \subseteq K \times W$.

Theorem 3.2. Let $\mathcal{W}$ be a covering of $Z$ and $G: \Delta^{k} \times Y \rightarrow Z$ be a $s t^{k}(\mathcal{W})$-continuous function and $s t^{k-1}(\mathcal{W})$ - continuous on $\partial \Delta^{k} \times Y$. Then there exists $\mathcal{V}$, a covering of $Y$, such that for each function $f: X \rightarrow Y$ that is $\mathcal{V}$-continuous, $G(i d \times f): \Delta^{k} \times X \rightarrow Z$ is $s t^{k}(\mathcal{W})$-continuous, and $G(i d \times f)$ is $s t^{k-1}(\mathcal{W})$ - continuous on $\partial \Delta^{k} \times X$. 
Proof. Let $y \in Y$ be a fixed point. For each $s \in \Delta^{k} \backslash \partial \Delta^{k}$, there exists $J_{\underline{s}} \subseteq \Delta^{k} \backslash \partial \Delta^{k}$, a neighborhood of $\underline{s}$, and a neighborhood $V_{\bar{y}}^{\underline{s}}$ of $y$, so that $\left.\underline{G\left(J_{\underline{s}}\right.} \times V_{\bar{y}}^{\underline{s}}\right) \subseteq W^{\underline{s}}$, for some element $W^{\underline{s}} \in s t^{k}(\mathcal{W})$. For each $\underline{s} \in \partial \Delta^{k}$, there exists $J_{\underline{s}} \subseteq N$, a neighborhood of $\underline{s}$, and a neighborhood $V_{\bar{y}}^{\underline{s}}$ of $y$, so that $G\left(J_{\underline{s}} \times \bar{V} \frac{s}{y}\right) \subseteq W \frac{s}{y}$, for some element $W^{\underline{s}} \in s t^{k-1}(\mathcal{W})$.

Then $\left\{J_{\underline{s}} \mid \underline{s} \in \Delta^{k}\right\}$ is an open covering of $\Delta^{k}$. There exists a finite subcovering $J_{\underline{s_{1}}}, J_{\underline{s_{2}}}, \ldots, J_{\underline{s_{p}}}$.

Let $\overline{J_{\underline{s}} y}=V_{\bar{y}}^{\underline{s_{1}}} \cap \ldots V_{\bar{y}} \underline{s_{p}}$. Then $G\left(J_{\underline{s}_{i}} \times V_{y}\right) \subseteq W_{*} \frac{s_{i}}{y}$, for $\underline{s}_{i} \in \Delta^{k} \backslash \partial \Delta^{k}$, and $G\left(J_{\underline{s}_{i}} \times V_{y}\right) \subseteq W \frac{s_{i}}{y}$, for $\underline{s}_{i} \in \partial \Delta^{k}$. $\mathcal{V}=\left\{V_{y} \mid y \in Y\right\}$ is a covering of $Y$. Let $V \in \mathcal{V}$. Then the following holds: $G\left(J_{\underline{s}_{i}} \times V_{y}\right) \subseteq W_{*} \frac{s_{i}}{y}$, for $\underline{s}_{i} \in \Delta^{k} \backslash \partial \Delta^{k}$, and $G\left(J_{\underline{s}_{i}} \times V_{y}\right) \subseteq W \frac{s_{i}}{y}$, for $\underline{s}_{i} \in \partial \Delta^{k} . J_{\underline{s}_{i}} \times V \mid i=1, \ldots, p, V \in \mathcal{V}$ is a covering of $\Delta^{k} \times Y$. If $f: X \rightarrow Y$ is a $\mathcal{V}$ - continuous function, then there exists a covering $\mathfrak{V}$ of $X$, so that $f(\mathfrak{V}) \prec \mathcal{V}$. Now, if we define $H: \Delta^{k} \times X \rightarrow Z$ by:

$$
H(\underline{t}, x)=G(\underline{t}, f(x)),
$$

then $H$ is a $s t^{k}(\mathcal{W})$ - continuous function and $H$ is $s t^{k-1}(\mathcal{W})$ - continuous on $\partial \Delta^{k} \times X$.

We will now define a partitioning of the simplex

$$
\Delta^{n}=\left\{\left(t_{1}, t_{2}, \ldots, t_{n}\right) \mid 1 \geq t_{1} \geq \ldots \geq t_{n} \geq 0\right\},
$$

by defining the sets $K_{m}, 0 \leq m \leq n$ in the following way:

$$
K_{m}=\left\{\left(t_{1}, \ldots, t_{n}\right) \mid t_{m} \geq \frac{1}{2} \geq t_{m+1}\right\} .
$$

Let $B$ be the denotation of the finite sets of coverings of $Y$ with a maximal element and $C$ be the denotation of the finite sets of coverings of $Z$ with a maximal element. Let $f=\left(f_{\underline{b}}\right): X \rightarrow Y$ and $g=\left(g_{c}\right): Y \rightarrow Z$ be coherent proximate nets. In order to proceed and define the composition $\underline{h}=\left(h_{\underline{c}}\right): X \rightarrow Z$ of $\underline{f}$ and $\underline{g}$, an induction by the height of the element $c \in C$ is performed.

Definition 3.3. Let $c \in C, h(c)=0$ be an ordered cofinite set. Then the height of a is defined as follows:

$$
h(a)=\max \left\{n \mid a_{0}<a_{1}<\cdots<a_{n-1}<a\right\} .
$$

A strictly increasing function $g: C \rightarrow B$ is constructed as follows:

Case 0. Let $c \in C, h(c)=1$. We choose an element $g(c)$, such that $g($ st $\max b) \prec \max c$. Let $g(c)=b$. Now $h_{c}: X \rightarrow Z$ may be defined by $h_{c}=g_{c} f_{g(c)}$. Then $h_{c}$ is $\max c$-continuous.

Case 1. Let $c \in C, h(c)=1$. We define $g(c)$, choosing $b \in B$, so that the following conditions hold:

1. $g($ st $\max b) \subseteq \max c$;

2. $g\left(c_{0}\right)<b$, for all possible choices of $c_{0}, c_{0}<c$; 
3. $g_{c_{0} c}\left(i d \times f_{b}\right)$ is st $\max c$-continuous and $g_{c_{0} c}\left(i d \times f_{b}\right)$ is $\max c$ continuous on $\partial \Delta^{1} \times X$.

Let $g(c)=b$. The functions $h_{c}$ and $h_{c_{0} c}$ are defined as follows:

$$
h_{c}=g_{c} f_{g(c)} \text {. }
$$

Then $h_{c}$ is $\max c$ - continuous. The function $h_{c_{0} c}: \Delta^{1} \times X \rightarrow Z$ is defined by:

$$
h_{c_{0} c}(t, x)=\left\{\begin{array}{ll}
g_{c_{0} c}\left(2 t-1, f_{b}(x)\right), & t \in K_{0} \\
g_{c_{0}} f_{b_{0} b}(2 t, x), & t \in K_{1}
\end{array} .\right.
$$

Theorem 3.1 provides that $g_{c_{0}} f_{b_{0} b}$ is $s t \max c_{0^{-}}$continuous, and Theorem 2.2 and the condition 3 provide that $g_{c_{0} c}\left(i d \times f_{b}\right)$ is st max $c_{0^{-}}$continuous and $g_{c_{0} c}\left(i d \times f_{b}\right)$ is $\operatorname{maxc}_{0^{-}}$continuous on $\partial \Delta^{1} \times X$. Then $h_{c_{0} c}$ is $s t \max c_{0^{-}}$continuous and $h_{c_{0} c}$ is $\max c_{0^{-}}$continuous on $\partial \Delta^{1} \times X$. On Figure 1 below there is a given review of the mapping $h_{c_{0} c}$.

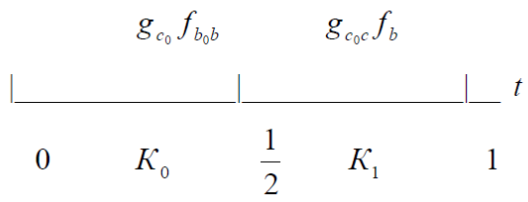

Figure 1

Case $n-1$ (inductive assumption). We assume that for each $c$ having a height $h(c) \leq n-1, g(c)=b$ is defined, so that the following conditions hold:

1) $g($ st $\max b) \prec \max c$;

2) $g\left(c_{0}\right)<g\left(c_{1}\right)<\cdots<g\left(c_{n-2}\right)<g(c)=b$, for all possible choices of indices $C_{0}<c_{1}<\cdots<c_{n-2}<c$;

3) The following mappings are defined: $h_{c}, h_{c_{0} c}, h_{c_{0} c_{1} c}, \ldots, h_{c_{0} c_{1} \ldots c_{n-2} c}$, $0 \leq i \leq n-2$, such that $h_{c_{0} c_{1} \ldots c_{i} c}: \Delta^{i+1} \times X \rightarrow Z$ is $s t^{i+1} \max c_{0^{-}}$ continuous and $h_{c_{0} c_{1} \ldots c_{i} c}$ is $s t^{i} \max c_{0^{-}}$continuous on $\partial \Delta^{i+1} \times X$.

On Figure 2 below there is a given review of the mapping $h_{c_{0} c_{1} c}$, and the conditions 1-3 below hold.

1. $g($ st $\max b) \prec \max c$;

2. $g\left(c_{0}\right)<b$, for all possible choices of $c_{0}, c_{0}<c$;

3. $g_{c_{0} c}\left(i d \times f_{b}\right)$ is st max $c$ - continuous and $g_{c_{0} c}\left(i d \times f_{b}\right)$ is max $c$ - continuous on $\partial \Delta^{1} \times X$.

Case $n$. Let $c \in C, h(c)=n$. Herein it is important to mention that there exists a linear homeomorphism between the sets $K_{i}$ and $\Delta^{i} \times \Delta^{n-i}$ mapping vertices to vertices. We choose $b$, so that:

1. $g($ st $\max b) \prec \max c$; 


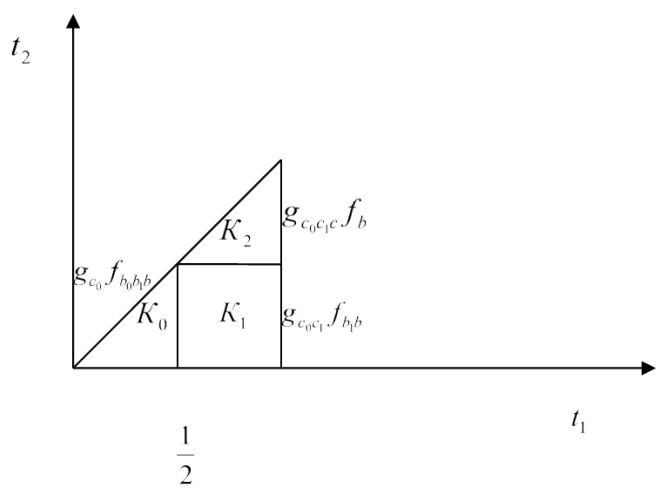

FiguRe 2

2. $g\left(c^{\prime}\right)<b$, for all possible choices of $c^{\prime}, c^{\prime}<c$;

3. $g_{c_{0} \ldots c_{k}}\left(i d \times f_{b_{k+1} \ldots b_{n}}\right): \Delta^{k} \times \Delta^{n-k} \times X \rightarrow Z$ is $s t^{k} \max c$ - continuous, for $k=1,2, \ldots n$ and for $\max b, g_{c_{0} \ldots c_{k}}\left(i d \times f_{b_{k+1} \ldots b_{n}}\right)$ is $s t^{k-1} \max c$ continuous on $\partial \Delta^{k} \times \Delta^{n-k} \times X$.

Let $g(c)=b$. Because of the inductive assumption, the following holds: $g\left(c_{0}\right)<g\left(c_{1}\right)<\cdots<g\left(c_{n-1}\right)<g(c)=b$ for each $c_{0}<c_{1}<\cdots<c_{n-2}<$ $c_{n-1}<c$, and the following mappings: $h_{c}, h_{c_{0} c}, h_{c_{0} c_{1} c}, \ldots, h_{c_{0} c_{1} \ldots c_{n-2} c}$ are defined. We define the function $h_{c_{0} c_{1} \ldots c_{n-1} c}: \Delta^{n} \times X \rightarrow Z$ by:

$$
h_{c_{0} c_{1} \ldots c_{n-1} c}(\underline{t}, x)=
$$

$\begin{cases}g_{c_{0}} f_{g\left(c_{0} c_{1} \ldots c_{n-1} c\right)}\left(2 t_{1}, \ldots, 2 t_{n}, x\right), & t_{1} \leq \frac{1}{2} \\ g_{c_{0} \ldots c_{i}}\left(2 t-1, \ldots, 2 t_{i}-1, f_{g\left(c_{i} c_{i+1} \ldots c_{n-1} c\right)}\left(2 t_{i+1}, \ldots, 2 t_{n}, x\right)\right), & t_{i} \geq \frac{1}{2} \geq t_{i+1} . \\ g_{c_{0} c_{1} \ldots c_{n-1} c}\left(2 t_{1}-1, \ldots, 2 t_{i}-1, f_{g(c)}(x)\right), & t_{n} \geq \frac{1}{2}\end{cases}$

Theorem 3.1, Theorem 3.2 and the condition 3 provide that $h_{c_{0} c_{1} \ldots c_{n-1} c}$ is $s t^{n} \max c_{0^{-}}$continuous and $h_{c_{0} c_{1} \ldots c_{n-1} c}$ is $s t^{n-1} \max c_{0^{-}}$continuous on $\partial \Delta^{n} \times$ $X$. We check that $h_{c_{0} c_{1} \ldots c_{n-1} c}$ is well defined. As in previous cases, $g\left(c_{0}\right)=$ $b_{0}, g\left(c_{1}\right)=b_{1}, \ldots, g\left(c_{n-1}\right)=b_{n-1}, g(c)=b$. Let $t_{1}=\frac{1}{2}$.

$$
\begin{aligned}
g_{c_{0}} f_{b_{0} b_{1} \ldots b_{n-1} b}\left(2 \cdot \frac{1}{2}, 2 t_{2}, \ldots, 2 t_{n}, x\right) & =g_{c_{0}} f_{b_{0} b_{1} \ldots b_{n-1} b}\left(1,2 t_{2}, \ldots, 2 t_{n}, x\right) \\
& =g_{c_{0}} f_{b_{0} b_{1} \ldots b_{n-1} b}\left(2 t_{2}, \ldots, 2 t_{n}, x\right) . \\
g_{c_{0} c_{1}}\left(2 \cdot \frac{1}{2}, f_{b_{0} b_{1} \ldots b_{n-1} b}\left(2 t_{2}, \ldots, 2 t_{n}, x\right)\right) & =g_{c_{0} c_{1}}\left(0, f_{b_{1} \ldots b_{n-1} b}\left(2 t_{2}, \ldots, 2 t_{n}, x\right)\right) \\
& =g_{c_{0}}\left(f_{b_{1} \ldots b_{n-1} b}\left(2 t_{2}, \ldots, 2 t_{n}, x\right)\right) \\
& =g_{c_{0}}\left(f_{b_{1} \ldots b_{n-1} b}\left(2 t_{2}, \ldots, 2 t_{n}, x\right)\right) .
\end{aligned}
$$


Let $t_{i}=t_{i+1}=\frac{1}{2} \cdot t_{i}=t_{i+1}$ implicates the following:

$$
\begin{aligned}
& g_{c_{0} \ldots c_{i}}\left(2 t_{1}-1,2 t_{2}-1, \ldots, 2 t_{i}-1, f_{b_{i} b_{i+1} \ldots b_{n-1} b}\left(2 t_{i+1}, \ldots, 2 t_{n}, x\right)\right) \\
& \quad=g_{c_{0} \ldots c_{i-1}}\left(2 t_{1}-1,2 t_{2}-1, \ldots, 2 t_{i}-1, f_{b_{i} b_{i+1} \ldots b_{n-1} b}\left(2 t_{i+1}, \ldots, 2 t_{n}, x\right)\right),
\end{aligned}
$$

whereas $t_{i}=\frac{1}{2}$ implicates:

$$
\begin{aligned}
& g_{c_{0} \ldots c_{i}}\left(2 t_{1}-1,2 t_{2}-1, \ldots, 2 t_{i}-1, f_{b_{i} b_{i+1} \ldots b_{n-1} b}\left(2 t_{i+1}, \ldots, 2 t_{n}, x\right)\right) \\
& \quad=g_{c_{0} \ldots c_{i}}\left(2 t_{1}-1,2 t_{2}-1, \ldots, 2 \frac{1}{2}-1, f_{b_{i} b_{i+1} \ldots b_{n-1} b}\left(2 t_{i}, \ldots, 2 t_{n}, x\right)\right) \\
& =g_{c_{0} \ldots c_{i}}\left(2 t_{1}-1,2 t_{2}-1, \ldots, 0, f_{b_{i} b_{i+1} \ldots b_{n-1} b}\left(2 t_{i+1}, \ldots, 2 t_{n}, x\right)\right) \\
& =g_{c_{0} \ldots c_{i-1}}\left(2 t_{1}-1,2 t_{2}-1, \ldots, 2 t_{i-1}-1, f_{b_{i} b_{i+1} \ldots b_{n-1} b}\left(2 t_{i+1}, \ldots, 2 t_{n}, x\right)\right) .
\end{aligned}
$$

Let $t_{n}=\frac{1}{2}$.

$$
\begin{gathered}
g_{c_{0} \ldots c_{n-1} c}\left(2 t_{1}-1,2 t_{2}-1, \ldots, 2 t_{n}-1\right) \\
=g_{c_{0} \ldots c_{n-1} c}\left(2 t_{1}-1,2 t_{2}-1, \ldots, 2 \frac{1}{2}-1, f_{b}(x)\right), \\
g_{c_{0} \ldots c_{n-1} c}\left(2 t_{1}-1,2 t_{2}-1, \ldots, 0, f_{b}(x)\right) \\
=g_{c_{0} \ldots c_{n-1}}\left(2 t_{1}-1,2 t_{2}-1, \ldots, 2 t_{n-1}-1, f_{b}(x)\right) . \\
g_{c_{0} \ldots c_{i}}\left(2 t_{1}-1,2 t_{2}-1, \ldots, 2 t_{i}-1, f_{b_{i} b_{i+1} \ldots b_{n-1} b}\left(2 t_{i+1}, \ldots, 2 t_{n}, x\right)\right) \\
=g_{c_{0} \ldots c_{n-1}}\left(2 t_{1}-1,2 t_{2}-1, \ldots, 2 t_{n}-1, f_{b_{n-1} b}\left(2 \cdot \frac{1}{2}, x\right)\right) \\
=g_{c_{0} \ldots c_{n-1}}\left(2 t_{1}-1,2 t_{2}-1, \ldots, 2 t_{n}-1, f_{b_{n-1} b}(1, x)\right) \\
=g_{c_{0} \ldots c_{n-1}}\left(2 t_{1}-1,2 t_{2}-1, \ldots, 2 t_{n}-1, f_{b}(x)\right) .
\end{gathered}
$$

Therefore it is obtained that the function is well defined. Consequently it is obtained that the composition of the coherent proximate nets $\underline{f}=\left(f_{\underline{b}}\right): X \rightarrow$ $Y$ and $\underline{g}=\left(g_{\underline{b}}\right): Y \rightarrow Z$ is a coherent proximate net $\underline{h}=\left(h_{\underline{c}}\right): Y \rightarrow Z$. Let this composition be denoted by $\underline{f} \circ \underline{g}: X \rightarrow Y$.

\section{Composition of homotopy Classes of COHEREnt PRoximate nets}

We define a composition of homotopy classes of coherent proximate nets by $\left[\left(g_{c}\right)\right]\left[\left(f_{b}\right)\right]=\left[\left(g_{c} f_{g(c)}\right)\right]$. In order for this definition to be valid, it is necessary to be proved that it does not depend on the choice of strictly increasing function $g: C \rightarrow B$. It is enough to show that for another choice of a strictly increasing function $g^{\prime}: C \rightarrow B$, such that it satisfies the same required conditions $1-3$ from the definition of composition in 2, the corresponding coherent proximate net $\underline{h}^{\prime}=\left(h_{\underline{c}}^{\prime}\right): X \rightarrow Z$ is in the same homotopy class with $\underline{h}=\left(h_{\underline{c}}\right): X \rightarrow Z$. In fact, as the way $g: C \rightarrow B$, and $\left(h_{\underline{c}}\right): X \rightarrow Z$, and $g^{\prime}: C \rightarrow B,\left(h_{\underline{c}}^{\prime}\right): X \rightarrow Z$ are obtained, similarly we may obtain another strictly increasing function $g^{\prime \prime}: C \rightarrow B$, with additional condition $g^{\prime \prime}(c)>g(c), g^{\prime}(c)$ for all $c \in C$, and a coherent proximate net $\left(h_{\underline{c}}^{\prime \prime}\right): X \rightarrow Z$. 
Now, by induction, a homotopy $\underline{H}=\left(H_{\underline{c}}\right): X \times I \rightarrow Z$, connecting $\left(h_{\underline{c}}\right)$ and $\left(h_{c}^{\prime \prime}\right)$, is constructed.

Case 0. If $c \in C, h(c)=1, H_{c}: I \times X \rightarrow Z$, is defined by $H_{c}(t, x)=$ $g_{c} f_{b g(c ")}(t, x)$. This homotopy connects $h_{c}=g_{c} f_{g(c)}$ and $h^{\prime \prime}=g_{c} f_{g(c ")}$.

Case 1. Let $c \in C, h(c)=1$. The homotopy $H_{c}: I \times X \rightarrow Z$ is defined by $H_{c}(t, x)=g_{c} f_{b g\left(c^{\prime \prime}\right)}(t, x)$. The following step is to show that $h_{c_{0} c}$ is homotopic to $h^{\prime \prime} c_{0} c$. Therefore, $H_{c_{0} c}: \Delta^{1} \times I \times X \rightarrow Z$ is defined in the following way (Figure 3):

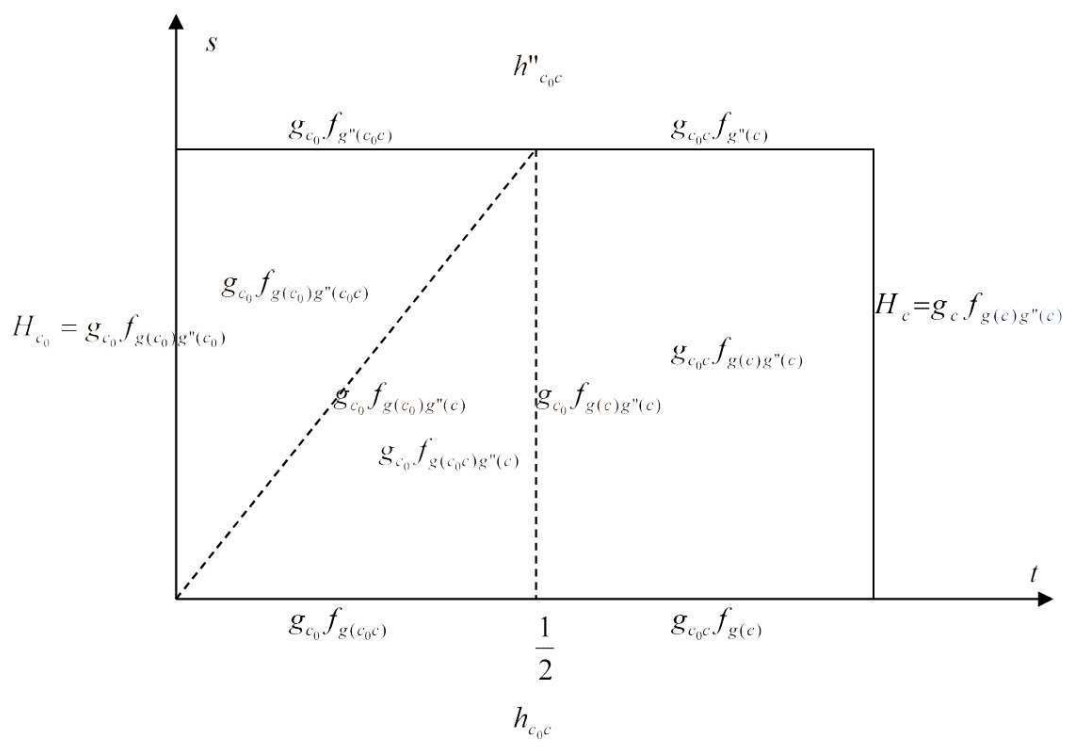

FIGURE 3

$$
H_{c_{0} c}(t, s, x)=\left\{\begin{array}{ll}
g_{c_{0}} f_{g\left(c_{0}\right) g^{\prime \prime}\left(c_{0} c\right)}(s, 2 t, x), & 0 \leq t \leq \frac{s}{2} \\
g_{c_{0}} f_{g\left(c_{0} c\right) g "(c)}(s, 2 t, x), & \frac{s}{2} \leq t \leq \frac{1}{2} \\
g_{c_{0} c}\left(2 t-1, f_{g(c) g "(c)}(s, x)\right), & \frac{1}{2} \leq t \leq 1
\end{array} .\right.
$$

$H_{c_{0} c}$ is well defined on the edges, and it is shown as follows: - If $t=\frac{s}{2}$, then

$$
g_{c_{0}} f_{b_{0} b_{0} " b "}\left(s, 2 \cdot \frac{s}{2}, x\right)=g_{c_{0}} f_{b_{0} b_{0} " b "}(s, s, x)=g_{c_{0}} f_{b_{0} b "}(s, x) .
$$

On the other hand,

$$
g_{c_{0}} f_{b_{0} b b},\left(2 \cdot \frac{s}{2}, s, x\right)=g_{c_{0}} f_{b_{0} b b^{\prime \prime}}(s, s, x)=g_{c_{0}} f_{b_{0} b "}(s, x) .
$$


If $t=\frac{1}{2}$, then

$$
g_{c_{0}} f_{b_{0} b b},\left(2 \cdot \frac{1}{2}, s, x\right)=g_{c_{0}} f_{b_{0} b b},(1, s, x)=g_{c_{0}} f_{b b} "(s, x) .
$$

On the other hand,

$$
g_{c_{0} c}\left(2 \cdot \frac{1}{2}-1, f_{b b "}(s, x)\right)=g_{c_{0} c}\left(0, f_{b b} "(s, x)\right)=g_{c_{0}} f_{b b "}(s, x) .
$$

The following also holds: If $s=0$, then

$$
\begin{aligned}
& H_{c_{0} c}(t, 0, x)= \begin{cases}g_{c_{0}} f_{b_{0} b_{0} "}{ }^{\prime \prime},(0,2 t, x), & t=0 \\
g_{c_{0}} f_{b_{0} b b "}(2 t, 0, x), & 0 \leq t \leq \frac{1}{2} \\
g_{c_{0} c}\left(2 t-1, f_{b b},(0, x)\right), & \frac{1}{2} \leq t \leq 1\end{cases} \\
& = \begin{cases}g_{c_{0}} f_{b_{0} b_{0}} " b^{\prime \prime}(0,0, x), & t=0 \\
g_{c_{0}} f_{b_{0} b b "}(2 t, 0, x), & 0 \leq t \leq \frac{1}{2} \\
g_{c_{0} c}\left(2 t-1, f_{b b},(0, x)\right), & \frac{1}{2} \leq t \leq 1\end{cases} \\
& = \begin{cases}g_{c_{0}} f_{b_{0} b_{0}} "(0, x), & t=0 \\
g_{c_{0}} f_{b_{0} b}(2 t, x), & 0 \leq t \leq \frac{1}{2} \\
g_{c_{0} c}\left(2 t-1, f_{b}(x)\right), & \frac{1}{2} \leq t \leq 1\end{cases} \\
& = \begin{cases}g_{c_{0}} f_{b_{0}}(x), & t=0 \\
g_{c_{0}} f_{b_{0} b}(2 t, x), & 0 \leq t \leq \frac{1}{2} \\
g_{c_{0} c}\left(2 t-1, f_{b}(x)\right), & \frac{1}{2} \leq t \leq 1\end{cases} \\
& =\left\{\begin{array}{ll}
g_{c_{0}} f_{b_{0} b}(2 t, x), & 0 \leq t \leq \frac{1}{2} \\
g_{c_{0} c}\left(2 t-1, f_{b}(x)\right), & \frac{1}{2} \leq t \leq 1
\end{array}=h_{c_{0} c}(t, x) .\right.
\end{aligned}
$$

If $s=1$, then

$$
\begin{aligned}
& H_{c_{0} c}(t, 1, x)= \begin{cases}g_{c_{0}} f_{b_{0} b_{0} "} b^{\prime \prime}(1,2 t, x), & 0 \leq t \leq \frac{1}{2} \\
g_{c_{0}} f_{b_{0} b b},(2 t, 1, x), & t=\frac{1}{2} \\
g_{c_{0} c}\left(2 t-1, f_{b b},(1, x)\right), & \frac{1}{2} \leq t \leq 1\end{cases} \\
& = \begin{cases}g_{c_{0}} f_{b_{0} b_{0} " b^{\prime}},(1,2 t, x), & 0 \leq t \leq \frac{1}{2} \\
g_{c_{0}} f_{b_{0} b b^{\prime \prime}}(1,1, x), & t=\frac{1}{2} \\
g_{c_{0} c}\left(2 t-1, f_{b b},(1, x)\right), & \frac{1}{2} \leq t \leq 1\end{cases} \\
& = \begin{cases}g_{c_{0}} f_{b_{0} b_{0} " b^{\prime \prime}}(1,2 t, x), & 0 \leq t \leq \frac{1}{2} \\
g_{c_{0}} f_{b b "}(1, x), & t=\frac{1}{2} \\
g_{c_{0} c}\left(2 t-1, f_{b},(x)\right), & \frac{1}{2} \leq t \leq 1\end{cases} \\
& = \begin{cases}g_{c_{0}} f_{b_{0} "} ",(2 t, x), & 0 \leq t \leq \frac{1}{2} \\
g_{c_{0}} f_{b "}(x), & t=\frac{1}{2} \\
g_{c_{0} c}\left(2 t-1, f_{b},(x)\right), & \frac{1}{2} \leq t \leq 1\end{cases} \\
& =\left\{\begin{array}{ll}
g_{c_{0}} f_{b_{0} " b^{\prime \prime}}(2 t, x), & 0 \leq t \leq \frac{1}{2} \\
g_{c_{0} c}\left(2 t-1, f_{b},(x)\right), & \frac{1}{2} \leq t \leq 1
\end{array}=h^{\prime \prime}{ }_{c_{0} c}(t, x) .\right.
\end{aligned}
$$

Hence it is proved that $H_{c_{0} c}$ is a homotopy between $h_{c_{0} c}$ and $h "{ }_{c_{0} c}$. 
Case $n$. The homotopy $H_{c_{0} c_{1} \ldots c_{n-1} c}: \Delta^{n} \times I \times X \rightarrow Z$ is defined in the following way: We have defined the partitioning of the non-standard simplex $\Delta^{n}=\left\{\left(t_{1}, t_{2}, \ldots, t_{n}\right) \mid 1 \geq t_{1} \geq t_{2} \ldots \geq t_{n} \geq 0\right\}$ by the sets $K_{i}, 0 \leq i \leq n$ where $K_{i}=\left\{\left(t_{1}, t_{2}, \ldots, t_{n}\right) \mid t_{i} \geq \frac{1}{2} \geq t_{i+1}\right\}$. Now we need a partitioning of the sets, and therefore we define the sets for each, in the following way:

$$
\begin{aligned}
K_{i}^{j}= & \left\{\left(t_{1}, \ldots, t_{i}, \ldots, t_{n}, s\right) \mid\left(t_{1}, \ldots, t_{i}, \ldots, t_{n}, s\right) \in K_{i} \times I\right. \\
& \text { so that } \left.\forall m, i \leq m \leq n-j, \frac{s}{2} \leq t_{m} \leq \frac{1}{2}, \forall l, n-j<l \leq n, 0 \leq t_{l} \leq \frac{1}{2}\right\} .
\end{aligned}
$$

Now $H_{c_{0} c_{1} \ldots c_{n-1} c}: \Delta^{n} \times I \times X \rightarrow Z$ is defined on $K_{i} \times I, \forall i=0,1, \ldots, n$, by:

$$
\begin{aligned}
H_{c_{0} c_{1} \ldots c_{n-1} c} & \left(t_{1}, \ldots, t_{n}, s, x\right) \\
= & g_{c_{0} c_{1} \ldots c_{i}}\left(2 t_{1}-1, \ldots, 2 t_{i}-1, f_{g\left(c_{i}, \ldots, c_{n-j}\right) g "\left(c_{n-j} c_{n-j+1} \ldots c\right)}\right. \\
& \left.\left(2 t_{i+1}, \ldots, s, 2 t_{n-j+1}, \ldots, 2 t_{n}, x\right)\right)
\end{aligned}
$$

for $\left(t_{1}, \ldots, t_{n}, s\right) \in K_{i}^{j}$. Next we show that $H_{c_{0} c_{1} \ldots c_{n-1} c}$ is well defined. In fact, we need to observe several cases, and for each we will show that $H_{c_{0} c_{1} \ldots c_{n-1} c}$ is well defined:

1. For $\left(t_{1}, \ldots, t_{n}, s, x\right) \in K_{i-1}^{j} \cap K_{i}^{j}, j=1, \ldots, n-i$, we will show that the definition of $H_{c_{0} c_{1} \ldots c_{n-1} c}$ is unique.

2. For $\left(t_{1}, \ldots, t_{n}, s, x\right) \in K_{i}^{j-1} \cap K_{i}^{j}, j=1, \ldots, n-i$, we will show that the definition of $H_{c_{0} c_{1} \ldots c_{n-1} c}$ is unique.

3. We will show that the definition of $H_{c_{0} c_{1} \ldots c_{n-1} c}$ on the edges of $\Delta^{n} \times I$ coincides with the corresponding homotopies on $\Delta^{n-1} \times I$, i.e., with $H_{c_{0} c_{1} \ldots \hat{c_{i} \ldots c_{n-1} c}}, i=1, \ldots, n$.

1. Let $\left(t_{1}, \ldots, t_{n}, s, x\right) \in K_{i-1}^{j} \cap K_{i}^{j}$, i.e. $t_{i}=\frac{1}{2}$. Because of $\left(t_{1}, \ldots, t_{n}, s, x\right)$ $\in K_{i-1}^{j}$ and $t_{i}=\frac{1}{2}$, we obtain the following:

$$
\begin{aligned}
& H_{c_{0} \ldots c}\left(t_{1}, \ldots, s, x\right) \\
& =g_{c_{0} \ldots c_{i-1}}\left(2 t_{1}-1, \ldots, 2 t_{i-1}-1\right. \text {, } \\
& \left.f_{b_{i-1} \ldots b_{n-j} " b_{n-j+1}} " \ldots b^{\prime \prime}\left(2 t_{i}, \ldots, s, 2 t_{n-j+1}, \ldots, 2 t_{n}, x\right)\right) \\
& =g_{c_{0} \ldots c_{i-1}}\left(2 t_{1}-1, \ldots, 2 t_{i-1}-1\right. \text {, } \\
& \left.f_{b_{i-1} \ldots b_{n-j}} " b_{n-j+1} " \ldots b "\left(2 \cdot \frac{1}{2}, \ldots, s, 2 t_{n-j+1}, \ldots, 2 t_{n}, x\right)\right) \\
& =g_{c_{0} \ldots c_{i-1}}\left(2 t_{1}-1, \ldots, 2 t_{i-1}-1\right. \text {, } \\
& \left.f_{b_{i-1} \ldots b_{n-j} " b_{n-j+1}} " \ldots b^{\prime \prime}\left(1, \ldots, s, 2 t_{n-j+1}, \ldots, 2 t_{n}, x\right)\right) \\
& =g_{c_{0} \ldots c_{i-1}}\left(2 t_{1}-1, \ldots, 2 t_{i-1}-1\right. \text {, } \\
& \left.f_{b_{i-1} \ldots b_{n-j} " b_{n-j+1} " \ldots b "}\left(2 t_{i+1}, \ldots, s, 2 t_{n-j+1}, \ldots, 2 t_{n}, x\right)\right) \text {. }
\end{aligned}
$$


On the other hand, because of $\left(t_{1}, \ldots, t_{n}, s, x\right) \in K_{i}^{j}$ and $t_{i}=\frac{1}{2}$, we obtain:

$$
\begin{aligned}
& H_{c_{0} \ldots c}\left(t_{1}, \ldots, s, x\right) \\
& =g_{c_{0} \ldots c_{i}}\left(2 t_{1}-1, \ldots, 2 \cdot \frac{1}{2}-1,\right. \\
& \left.f_{b_{i} \ldots b_{n-j}} " b_{n-j+1} " \ldots b^{\prime \prime}\left(2 t_{i+1}, \ldots, s, 2 t_{n-j+1}, \ldots, 2 t_{n}, x\right)\right) \\
& =g_{c_{0} \ldots c_{i-1}}\left(2 t_{1}-1, \ldots, 0\right. \text {, } \\
& \left.f_{b_{i} \ldots b_{n-j}} " b_{n-j+1} " \ldots b^{\prime \prime}\left(2 t_{i+1}, \ldots, s, 2 t_{n-j+1} \ldots, 2 t_{n}, x\right)\right) \\
& =g_{c_{0} \ldots c_{i-1}}\left(2 t_{1}-1, \ldots, 2 t_{i-1}-1\right. \text {, } \\
& \left.f_{b_{i} \ldots b_{n-j}} " b_{n-j+1} " \ldots b^{\prime \prime}\left(2 t_{i+1}, \ldots, s, 2 t_{n-j+1} \ldots, 2 t_{n}, x\right)\right)
\end{aligned}
$$

by which we show case 1 .

2. Let $\left(t_{1}, \ldots, t_{n}, s, x\right) \in K_{i}^{j-1} \cup K_{i}^{j}$, i.e. $t_{n-j+1}=\frac{s}{2}$. Because of $\left(t_{1}, \ldots, t_{n}, s, x\right) \in K_{i}^{j-1}$ and $t_{n-j+1}=\frac{s}{2}$, we obtain:

$$
\begin{aligned}
& H_{c_{0} \ldots c}\left(t_{1}, \ldots t_{n}, s, x\right) \\
& =g_{c_{0} \ldots c_{i}}\left(2 t_{1}-1, \ldots, 2 t_{i}-1,\right. \\
& \left.f_{b_{i} \ldots b_{n-j+1} b_{n-j+1}} " \ldots b^{\prime}\left(2 t_{i+1}, \ldots, 2 \cdot \frac{s}{2}, s, 2 t_{n-j+2}, \ldots, 2 t_{n}, x\right)\right) \\
& =c_{0} \ldots c_{i}\left(2 t_{1}-1, \ldots, 2 t_{i}-1\right. \text {, } \\
& \left.f_{b_{i} \ldots b_{n-j+1} b_{n-j+1} " \ldots b^{\prime \prime}}\left(2 t_{i+1}, \ldots, s, s, 2 t_{n-j+2}, \ldots, 2 t_{n}, x\right)\right) \\
& =g_{c_{0} \ldots c_{i}}\left(2 t_{1}-1, \ldots, 2 t_{i}-1\right. \text {, } \\
& \left.f_{b_{i} \ldots b_{n-j} b_{n-j+1}} " \ldots b^{\prime \prime}\left(2 t_{i+1}, \ldots, 2 t_{n-j}, s, 2 t_{n-j+2} \ldots, 2 t_{n}, x\right)\right) \text {. }
\end{aligned}
$$

On the other hand, because of $\left(t_{1}, \ldots, t_{n}, s, x\right) \in K_{i}^{j}$ and $t_{n-j+1}=\frac{s}{2}$, we obtain:

$$
\begin{aligned}
H_{c_{0} \ldots c} & \left(t_{1}, \ldots t_{n}, s, x\right) \\
= & g_{c_{0} \ldots c_{i}}\left(2 t_{1}-1, \ldots, 2 t_{i}-1\right. \\
& \left.f_{b_{i} \ldots b_{n-j+1} b_{n-j+1}}{ } \ldots b^{\prime \prime}\left(2 t_{i+1}, \ldots, \frac{2 s}{2}, s, 2 t_{n-j+2}, \ldots, 2 t_{n}, x\right)\right) \\
= & g_{c_{0} \ldots c_{i}}\left(2 t_{1}-1, \ldots, 2 t_{i}-1\right. \\
& \left.f_{b_{i} \ldots b_{n-j} b_{n-j} ", b_{n-j+1}, \ldots b^{\prime \prime}}\left(2 t_{i+1}, \ldots, s, \frac{2 s}{2}, 2 t_{n-j+2}, \ldots, 2 t_{n}, x\right)\right) \\
= & g_{c_{0} \ldots c_{i}}\left(2 t_{1}-1, \ldots, 2 t_{i}-1,\right. \\
& \left.f_{b_{i} \ldots b_{n-j} b_{n-j+1}}, \ldots b^{\prime \prime}\left(2 t_{i+1}, \ldots, 2 t_{n-j}, s, 2 t_{n-j+2} \ldots, 2 t_{n}, x\right)\right)
\end{aligned}
$$

by which case 2 is completed. 
3. The edges of $\Delta^{n} \times I$, because of the definition of $\Delta^{n}$, are of a type:

$$
\begin{aligned}
& \partial_{0}^{n}=\left\{\left(1, t_{2}, \ldots, t_{n}, s\right) \mid\left(1, t_{2}, \ldots, t_{n}\right) \in \Delta^{n}\right\} \\
& \partial_{l}^{n}=\left\{\left(1, t_{2}, \ldots, t_{n}, s\right) \mid\left(t_{1}, t_{2}, \ldots, t_{n}\right) \in \Delta^{n}, t_{l}=t_{l+1}\right\}, l=1 \ldots, n-1, \\
& \partial_{n}^{n}=\left\{\left(t_{1}, \ldots, t_{n-1}, 0, t, s\right) \mid\left(t_{1}, t_{2}, \ldots, t_{n-1}, 0\right) \in \Delta^{n}\right\} .
\end{aligned}
$$

On $\partial_{0}^{n}$ there are the sets $K_{1}, K_{2}, \ldots, K_{n}$. On $\partial_{l}^{n}$ there are the sets $K_{0}, \ldots, K_{i-1}, K_{i+1}, \ldots, K_{n}$. On $\partial_{n}^{n}$ there are the sets $K_{0}, K_{1}$, $\ldots, K_{n-1}$. For $\partial_{0}^{n}$, we obtain:

$$
\begin{aligned}
H_{c_{0} \ldots c} & \left(t_{1}, \ldots t_{n}, s, x\right) \\
= & g_{c_{0} \ldots c_{i}}\left(2 \cdot 1-1, \ldots, 2 t_{i}-1,\right. \\
& \left.f_{b_{i} \ldots b_{n-j} b_{n-j} " b_{n-j+1} " \ldots b "}\left(2 t_{i+1}, \ldots, s, 2 t_{n-j+1}, \ldots, 2 t_{n}, x\right)\right) \\
= & g_{c_{0} \ldots c_{i}}\left(1, \ldots, 2 t_{i}-1,\right. \\
& \left.f_{b_{i} \ldots b_{n-j} b_{n-j} ", b_{n-j+1} ", \ldots b "}\left(2 t_{i+1}, \ldots, s, 2 t_{n-j+1}, \ldots, 2 t_{n}, x\right)\right) \\
= & g_{c_{0} \ldots c_{i}}\left(2 t_{2}-1, \ldots, 2 t_{i}-1,\right. \\
& \left.f_{b_{i} \ldots b_{n-j} b_{n-j} " b_{n-j+1} " \ldots b "}\left(2 t_{i+1}, \ldots, s, 2 t_{n-j+1} \ldots, 2 t_{n}, x\right)\right) \\
= & H_{c_{1} \ldots c}\left(t_{2}, \ldots, t_{n}, s, x\right),
\end{aligned}
$$

for $\left(t_{2}, \ldots, t_{n}, s\right) \in K_{i}^{j}, \forall i=1, \ldots, n$.

For $\partial_{i}^{n}$, there are two cases, either $l<i$ or $l>i$ in the general formula of $H_{c_{0} c_{1} \ldots c_{n-1} c}$. It is impossible that $l=i$, because on the edge $\partial_{i}^{n}$ there is no set $K_{i}$. For $l<i$, we obtain:

$$
\begin{aligned}
H_{c_{0} \ldots c} & \left(t_{1}, \ldots t_{n}, s, x\right) \\
= & g_{c_{0} \ldots c_{i}}\left(2 t_{1}-1, \ldots, 2 t_{l}-1,2 t_{l+1}-1, \ldots 2 t_{i}-1,\right. \\
& \left.f_{b_{l} \ldots b_{n-j} b_{n-j} " \ldots b "}\left(2 t_{i+1}, \ldots, s, 2 t_{n-j+1}, \ldots, 2 t_{n}, x\right)\right) \\
= & g_{c_{0} \ldots c_{i}}\left(2 t_{1}-1, \ldots, 2 t_{l}-1,2 t_{i}-1,\right. \\
& \left.f_{b_{i} \ldots b_{n-j} b_{n-j} ", b_{n-j+1} ", \ldots b "}\left(2 t_{i+1}, \ldots, s, 2 t_{n-j+1}, \ldots, 2 t_{n}, x\right)\right) \\
= & g_{c_{0} \ldots \hat{c}_{l} \ldots c_{i}}\left(2 t_{2}-1, \ldots, 2 t_{i}-1,\right. \\
& \left.f_{b_{i} \ldots b_{n-j} b_{n-j} " b_{n-j+1}}{ } \ldots b^{\prime \prime}\left(2 t_{i+1}, \ldots, s, 2 t_{n-j+1}, \ldots, 2 t_{n}, x\right)\right) \\
= & H_{c_{0} c_{1} \ldots \hat{c}_{l} \ldots c_{n-1} c}\left(t_{1}, \ldots, \hat{t}_{l+1}, t_{l+2}, \ldots, t_{i}, \ldots, t_{n}, s, x\right),
\end{aligned}
$$

for $\left(t_{1}, \ldots, \hat{t}_{l+1}, t_{l+2}, \ldots, t_{i}, \ldots, t_{n}, s\right) \in K_{i}^{j}, i=1, \ldots, \hat{l}, \ldots, n$. For $l>i$, there are also two cases possible: either both $t_{l}$ and $t_{l+1}$ are before $s$, or both $t_{l}$ and $t_{l+1}$ are after $s$. We will show the case when both $t_{l}$ and $t_{l+1}$ are before 
$s$, and the other can be obtained similarly:

$$
\begin{aligned}
H_{c_{0} c_{1} \ldots c_{n-1} c}\left(t_{1}, \ldots t_{n}, s, x\right) & \\
= & g_{c_{0} \ldots c_{i}}\left(2 t_{1}-1, \ldots, 2 t_{i}-1,\right. \\
& \left.f_{b_{i} \ldots b_{n-j} b_{n-j} " \ldots b^{\prime \prime}}\left(2 t_{i+1}, \ldots, 2 t_{l}, 2 t_{l+1}, \ldots, s, 2 t_{n-j+1}, \ldots, 2 t_{n}, x\right)\right) \\
= & g_{c_{0} \ldots c_{i}}\left(2 t_{1}-1, \ldots, 2 t_{l}-1,2 t_{i}-1,\right. \\
& f_{b_{i} \ldots b_{n-j} b_{n-j} ", b_{n-j+1} ", \ldots b "} \\
& \left.\left(2 t_{i+1}, \ldots, 2 t_{l}, 2 t_{l}, \ldots, s, 2 t_{n-j+1}, \ldots, 2 t_{n}, x\right)\right) \\
= & g_{c_{0} \ldots c_{i}}\left(2 t_{1}-1, \ldots, 2 t_{i}-1,\right. \\
& f_{b_{i} \ldots \hat{b}_{l} \ldots b_{n-j} b_{n-j} " b_{n-j+1} " \ldots b^{\prime \prime}} \\
& \left.\left(2 t_{i+1}, \ldots, 2 t_{l}, 2 t_{l+2}, \ldots, s, 2 t_{n-j+1}, \ldots, 2 t_{n}, x\right)\right) \\
= & H_{c_{0} c_{1} \ldots \hat{c}_{l} \ldots c_{n-1} c}\left(t_{1}, \ldots, t_{i}, \ldots, \hat{t}_{l+1}, \ldots, t_{n}, s, x\right),
\end{aligned}
$$

for $\left(t_{1}, \ldots, t_{i}, \ldots \hat{t}_{l+1}, \ldots, t_{n}, s\right) \in K_{i}^{j}, i=1, \ldots, \hat{l}, \ldots, n$. For $\partial_{n}^{n}$, we obtain:

$$
\begin{aligned}
& H_{c_{0} c_{1} \ldots c_{n-1} c}\left(t_{1}, \ldots t_{n}, s, x\right) \\
&=g_{c_{0} \ldots c_{i}}\left(2 t_{1}-1, \ldots, 2 t_{i}-1\right. \\
&\left.f_{b_{i} \ldots b_{n-j} b_{n-j} " \ldots b "}\left(2 t_{i+1}, \ldots, s, 2 t_{l}, 2 t_{n-j+1}, \ldots, 0, x\right)\right) \\
&= g_{c_{0} \ldots c_{i}}\left(2 t_{1}-1, \ldots, 2 t_{i}-1\right. \\
&\left.f_{b_{i} \ldots b_{n-j} b_{n-j} ", b_{n-j+1} ", \ldots b "}\left(2 t_{i+1}, \ldots, s, 2 t_{n-j+1}, \ldots, 0, x\right)\right) \\
&= g_{c_{0} \ldots c_{i}}\left(2 t_{1}-1, \ldots, 2 t_{i}-1,\right. \\
&\left.f_{b_{i} \ldots b_{n-j} b_{n-j} " b_{n-j+1} " \ldots b^{\prime \prime}}\left(2 t_{i+1}, \ldots, s, 2 t_{n-j+1}, \ldots, 0, x\right)\right) \\
&= H_{c_{0} c_{1} \ldots c_{n-1}}\left(t_{1}, \ldots, t_{i}, \ldots, t_{n-1}, s, x\right),
\end{aligned}
$$

$\left(t_{1}, \ldots, t_{i}, \ldots, t_{n-1}, s\right) \in K_{i}^{j}, i=0, \ldots, n-1$. Showing case 3 is in fact showing the coherence condition for $H_{c_{0} c_{1} \ldots c_{n-1} c}$. We have also shown that the homotopy $H_{c_{0} c_{1} \ldots c_{n-1} c}$ is well defined. Next we show that $H_{c_{0} c_{1} \ldots c_{n-1} c}$ connects $h_{c_{0} c_{1} \ldots c_{n-1} c}$ and $h "{ }_{c_{0} c_{1} \ldots c_{n-1} c}$ : For $s=0$, by the definition of the sets $K_{i}^{j}$, it follows that for each $n-j<l \leq n, t_{l}=0$, and therefore we obtain:

$$
\begin{aligned}
& H_{c_{0} c_{1} \ldots c_{n-1} c}\left(t_{1}, \ldots t_{n}, 0, x\right) \\
&= g_{c_{0} \ldots c_{i}}\left(2 t_{1}-1, \ldots, 2 t_{i}-1,\right. \\
&\left.f_{b_{i} \ldots b_{n-j} b_{n-j} " b_{n-j+1} \ldots b^{\prime \prime}}\left(2 t_{i+1}, \ldots, s, 2 t_{l}, 2 t_{n-j+1}, 0, \ldots, 0, x\right)\right) \\
&= g_{c_{0} \ldots c_{i}}\left(2 t_{1}-1, \ldots, 2 t_{i}-1,\right. \\
&\left.f_{b_{i} \ldots b_{n-j}}\left(2 t_{i+1}, \ldots, s, 2 t_{n-j+1}, x\right)\right),
\end{aligned}
$$

for $\left(t_{1}, \ldots, t_{n}, 0\right) \in K_{i}^{j}$, which corresponds to the definition of $h_{c_{0} c_{1} \ldots c_{n-1} c}$. 


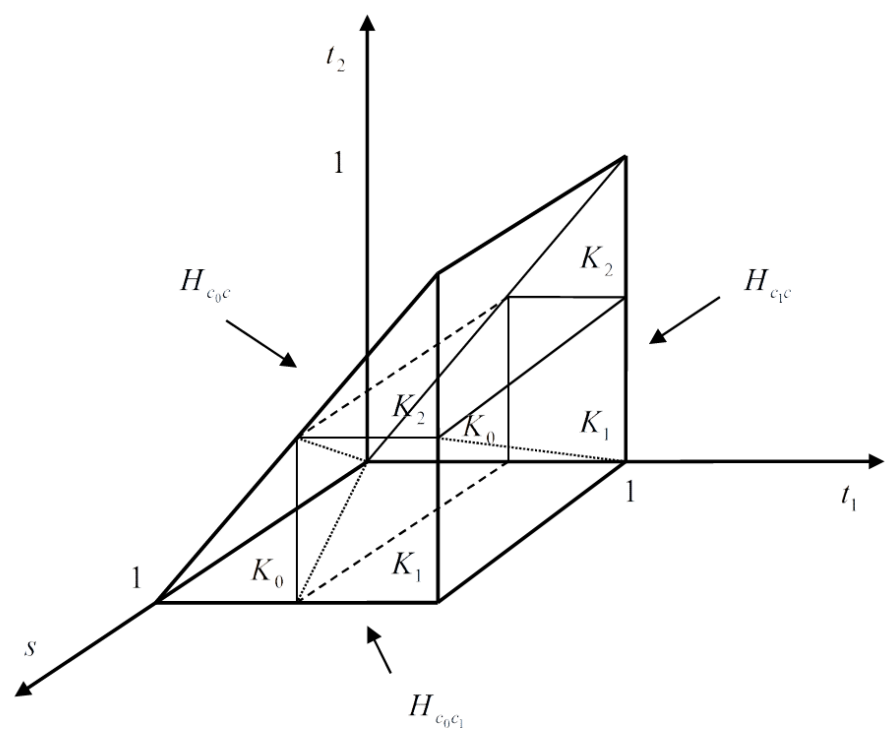

FiguRe 4

For $s=1$, by the definition of the sets $K_{i}^{j}$, it follows that $\forall m, i<m \leq$ $n-j, t_{m}=\frac{1}{2}$, and therefore we obtain:

$$
\begin{aligned}
& H_{c_{0} c_{1} \ldots c_{n-1} c}\left(t_{1}, \ldots t_{n}, 1, x\right)= \\
&= g_{c_{0} \ldots c_{i}}\left(2 t_{1}-1, \ldots, 2 t_{i}-1,\right. \\
&\left.f_{b_{i} \ldots b_{n-j} b_{n-j} " b_{n-j+1} \ldots b^{\prime \prime}}\left(2 \cdot \frac{1}{2}, \ldots, 2 \cdot \frac{1}{2}, 2 t_{n-j+1}, \ldots, x\right)\right) \\
&= g_{c_{0} \ldots c_{i}}\left(2 t_{1}-1, \ldots, 2 t_{i}-1,\right. \\
&\left.f_{b_{i} \ldots b_{n-j} b_{n-j} " b_{n-j+1} b^{\prime}}\left(1, \ldots, 1, s, 2 t_{n-j+1}, 2 t_{n}, x\right)\right) \\
&= g_{c_{0} \ldots c_{i}}\left(2 t_{1}-1, \ldots, 2 t_{i}-1\right. \\
& f_{b_{n-i}}{ }^{\prime \prime} \ldots b_{n-j+1} " \ldots b^{\prime \prime} \\
&
\end{aligned}
$$

for $\left(t_{1}, \ldots, t_{n}, 1\right) \in K_{i}^{j}$, which corresponds to the definition of $h^{\prime \prime} c_{0} c_{1} \ldots c_{n-1} c$. Next we observe the example when $n=2$, in order to illustrate the above. $h_{c_{0} c_{1} c}$ and $h_{c_{0} c_{1} c}$ " were previously defined in the following way (Figure 2):

$h_{c_{0} c_{1} c}\left(t_{1}, t_{2}, x\right)=\left\{\begin{array}{ll}g_{c_{0}} f_{b_{0} b_{1} b}\left(2 t_{1}, 2 t_{2}, x\right), & t_{1} \leq \frac{1}{2}\left(\left(t_{1}, t_{2}\right) \in K_{0}\right) \\ g_{c_{0} c_{1}}\left(2 t_{1}-1, f_{b_{1} b}\left(2 t_{2}, x\right)\right), & t_{1} \geq \frac{1}{2} \geq t_{2}\left(\left(t_{1}, t_{2}\right) \in K_{1}\right), \\ g_{c_{0} c_{1} c}\left(2 t_{1}, 2 t_{2}, f_{b}(x)\right), & t_{2} \leq \frac{1}{2}\left(\left(t_{1}, t_{2}\right) \in K_{2}\right)\end{array}\right.$, 
$h^{\prime \prime} c_{0} c_{1} c\left(t_{1}, t_{2}, x\right)= \begin{cases}g_{c_{0}} f_{b_{0} " b_{1} " b "}\left(2 t_{1}, 2 t_{2}, x\right), & t_{1} \leq \frac{1}{2}\left(\left(t_{1}, t_{2}\right) \in K_{0}\right) \\ g_{c_{0} c_{1}}\left(2 t_{1}-1, f_{b_{1}} " b "\left(2 t_{2}, x\right),\right) & t_{1} \geq \frac{1}{2} \geq t_{2}\left(\left(t_{1}, t_{2}\right) \in K_{1}\right) \\ g_{c_{0} c_{1} c}\left(2 t_{1}, 2 t_{2}, f_{b} "(x)\right), & t_{2} \leq \frac{1}{2}\left(\left(t_{1}, t_{2}\right) \in K_{2}\right)\end{cases}$

The homotopy $H_{c_{0} c_{1} c}: \Delta^{2} \times I \times X \rightarrow Z$, which connects $h_{c_{0} c_{1} c}$ and $h{ }^{\prime} c_{0} c_{1} c$, is defined on $K_{i} \times I$, for each $i=0,1,2$, by:

$$
\begin{aligned}
& H_{c_{0} c_{1} c}\left(t_{1}, t_{2}, s, x\right) \\
& \quad=g_{c_{0} \ldots c_{i}}\left(2 t_{1}-1, \ldots, 2 t_{i}-1, f_{b_{i} \ldots b_{2-j} b_{2-j}}{ } \ldots b^{\prime}\right. \\
&
\end{aligned}
$$

for $\left(t_{1}, t_{2}, s\right) \in K_{i}^{j}, j=0, \ldots, 2-i$. More specifically, $H_{c_{0} c_{1} c}$ is defined as follows, for each $K_{i} \times I, i=0,1,2$ : For $K_{0} \times I$ :

$$
H_{c_{0} c_{1} c}\left(t_{1}, t_{2}, s, x\right)=\left\{\begin{array}{ll}
g_{c_{0}} f_{b_{0} b_{1} b b "}\left(2 t_{1}, 2 t_{2}, s, x\right), & \left(t_{1}, t_{2}, s\right) \in K_{0}^{0} \\
g_{c_{0}} f_{b_{0} b_{1} b_{1} " b "}{ }^{\prime}\left(2 t_{1}, s, 2 t_{2}, x\right), & \left(t_{1}, t_{2}, s\right) \in K_{0}^{1} \\
g_{c_{0}} f_{b_{0} b_{0} " b_{1} " b "}{ }^{\prime}\left(s, 2 t_{1}, 2 t_{2}, x\right), & \left(t_{1}, t_{2}, s\right) \in K_{0}^{2}
\end{array} .\right.
$$

- For $K_{1} \times I$ :

$$
H_{c_{0} c_{1} c}\left(t_{1}, t_{2}, s, x\right)=\left\{\begin{array}{ll}
g_{c_{0} c_{1}}\left(2 t_{1}, f_{b_{1} b b "}\left(2 t_{2}, s, x\right)\right), & \left(t_{1}, t_{2}, s\right) \in K_{1}^{0} \\
g_{c_{0} c_{1}}\left(2 t_{1}, f_{b_{1} b_{1}} b^{\prime},\left(2 t_{2}, s, x\right)\right), & \left(t_{1}, t_{2}, s\right) \in K_{1}^{1}
\end{array} .\right.
$$

- For $K_{1} \times I$ :

$$
H_{c_{0} c_{1} c}\left(t_{1}, t_{2}, s, x\right)=g_{c_{0} c_{1} c}\left(2 t_{1}, 2 t_{2}-1, f_{b b},(s, x)\right),\left(t_{1}, t_{2}, s\right) \in K_{2}^{0} .
$$

On Figure 4 we see the way $\Delta^{2} \times I$ divides itself into three prisms $K_{0} \times I$, $K_{1} \times I$, and $K_{2} \times I$. On Figures 5, 6 and 7 below we can see the corresponding partitioning of $K_{i} \times I$ into $K_{i}^{j}, i=0,1,2 .-K_{0} \times I$ divides into three parts, $K_{0}^{0}, K_{0}^{1}$ and $K_{0}^{2}$ : $-K_{1} \times I$ divides into two parts, $K_{1}^{0}$ and $K_{1}^{1}$ : $-K_{2} \times I$ does not divide and the one part is $K_{2}^{0}$ :

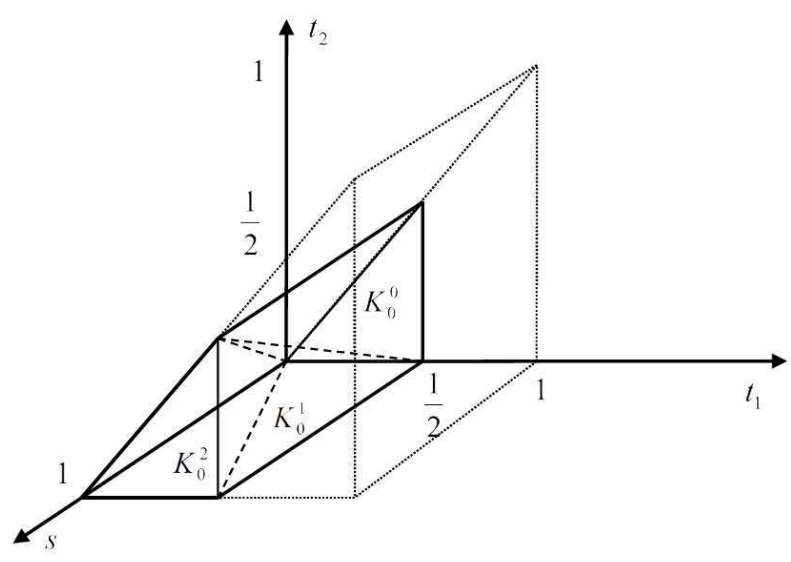

FIGURE 5 




Figure 6

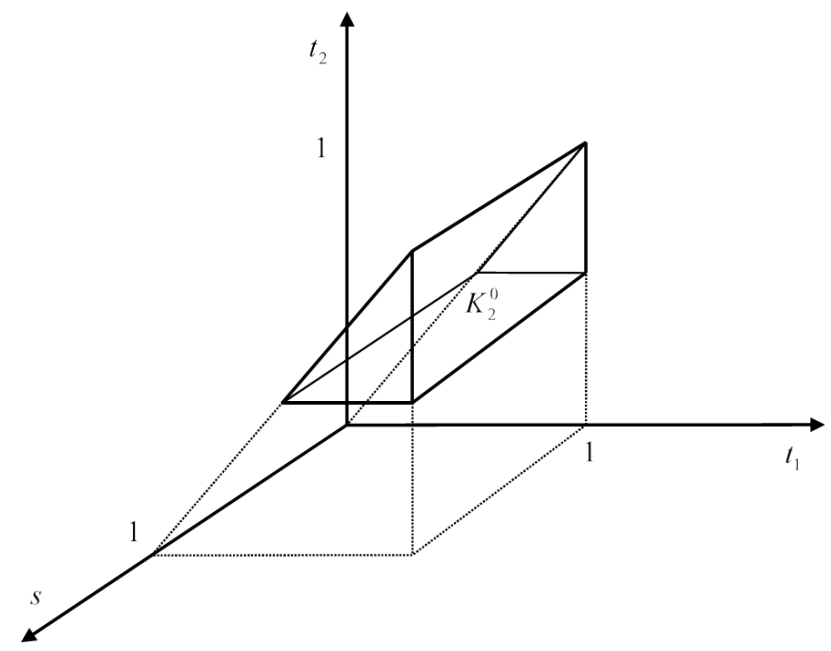

FiguRE 7

Hence it is shown that $\left(h_{c}\right)$ and $\left(h{ }_{c}\right)$ are homotopic. In an analogue way we prove that $\left(h_{c}\right)$ and $\left(h_{c}{ }_{c}\right)$ are homotopic. The relation of homotopy of coherent nets is an equivalence relation, therefore $\left(h_{c}\right)=\left(g_{c} f_{g(c)}\right)$ and $\left(h^{\prime \prime}{ }_{c}\right)=\left(g_{c} f_{g^{\prime}(c)}\right)$ and are in the same homotopy class. Now we may define a composition of homotopy classes of coherent proximate nets by

$$
\left[\left(g_{c}\right)\right]\left[\left(f_{b}\right)\right]=\left[\left(g_{c} f_{g(c)}\right)\right]
$$


and this definition does not depend on the choice of strictly increasing function $g: C \rightarrow B$.

\section{The CATEGORY OF STRONG SHAPE}

TheOREM 5.1. If $f: X \rightarrow Y, g: Y \rightarrow Z, h: Z \rightarrow W=\left(W_{d}, w_{\underline{d}}, D\right)$ are proximate coherent nets, then the proximate coherent nets $h(g f)$ and $(h g) f$ are homotopic.

Proof. Suppose $f=\left(f_{\underline{b}}\right), g=\left(g_{\underline{c}}\right)$, and $h=\left(h_{\underline{d}}\right)$. In order to obtain an explicit formula for the proximate coherent net $h(g \bar{f})$, we define a decomposition of $\Delta^{n}$ into subpolyhedra $K_{l, m}$ for any pair of integers $l, m$ such that $0 \leq l \leq m \leq n, K_{l, m}=\left\{\left(t_{1}, t_{2}, \ldots, t_{n}\right) \mid t_{l} \geq \frac{1}{2} \geq t_{l+1}, t_{m} \geq \frac{1}{4} \geq t_{m+1}\right\}$. By applying the composition formula twice, for $\left(t_{1}, \ldots, t_{n}\right) \in K_{l, m}$ we have

$$
\begin{aligned}
(h(g f) & )_{\underline{d}}(t, x) \\
= & h_{d_{0} \ldots d_{l}}\left(2 t_{1}-1, \ldots, 2 t_{l}-1, g_{h\left(d_{1} \ldots d_{m}\right)}\left(4_{l+1}-1, \ldots, 4 t_{m}-1,\right.\right. \\
& \left.\left.f_{g h\left(d_{m} \ldots d_{k}\right)}\left(4 t_{m+1}, \ldots, 4 t_{k}, x\right)\right)\right) .
\end{aligned}
$$

Similarly, to obtain an explicit formula for the proximate coherent net $(h g) f$, we define a decomposition of $\Delta^{n}$ into subpolyhedra $Q_{l, m}$ for any pair of integers $l, m$ such that $0 \leq l \leq m \leq n, Q_{l, m}=\left\{\left(t_{1}, \ldots, t_{n}\right) \mid t_{l} \geq \frac{3}{4} \geq t_{l+1}, t_{m} \geq\right.$ $\left.\frac{1}{2} \geq t_{m+1}\right\}$. Then, for $\left(t_{1}, \ldots, t_{k}\right) \in Q_{l, m}$ we have

$$
\begin{aligned}
(h(g f))_{\underline{d}}(t, x) & \\
= & h_{d_{0} \ldots d_{l}}\left(4 t_{1}-3, \ldots, 4 t_{l}-3, g_{h\left(d_{1} \ldots d_{m}\right)}\left(4_{l+1}-m, \ldots, 4 t_{m}-2,\right.\right. \\
& \left.\left.f_{g h\left(d_{m} \ldots d_{k}\right)}\left(2 t_{m+1}, \ldots, 2 t_{k}, x\right)\right)\right) .
\end{aligned}
$$

We define a partition of $\Delta^{n} \times I$ into subpolyhedra $M_{l, m}$ for any pair of integers $l, m$ such that $0 \leq l \leq m \leq n$,

$$
M_{l, m}=\left\{\left(t_{1}, \ldots, t_{n}, s\right) \mid t_{l} \geq \frac{2+s}{4} \geq t_{l+1}, t_{m} \geq \frac{1+s}{2} \geq t_{m+1}\right\} .
$$

We define a homotopy $H: I \times X \rightarrow W$ which connects $h(g f)$ and $(h g) f$. This map will be given by the function $f g h: D \rightarrow A$ and by the maps $H_{\underline{d}}: \Delta^{n} \times I \times X_{f g h\left(d_{n}\right)} \rightarrow W_{d_{0}}$ defined in the following way:

$$
\begin{aligned}
H_{\underline{d}}(t, s, x) & \\
= & h_{d_{0} \ldots d_{l}}\left(\frac{4 t_{1}-2-s}{2-s}, \ldots, \frac{4 t_{l}-2-s}{2-s},\right. \\
& \left.g_{h\left(d_{1} \ldots d_{m}\right)}\left(4 t_{l+1}-2, \ldots, 4 t_{m}-2, f_{g h\left(d_{m} \ldots d_{n}\right)\left(2 t_{m+1}, \ldots 2 t_{n}, x\right)}\right)\right) .
\end{aligned}
$$

We mention that $K_{l, m} \times 0=\left\{\left(t_{1}, \ldots, t_{n}, 0\right) \mid\left(t_{1}, \ldots, t_{n}, 0\right) \in M_{l, m}\right\}$ and then, for $\left(t_{1}, \ldots, t_{n}\right) \in K_{l, m}$, it is easily checked that $H_{\underline{d}}(t, 0, x)=(h(g f)) \frac{j}{d}(t, x)$. Also, $Q_{l, m} \times 1=\left\{\left(t_{1}, \ldots, t_{n}, 1\right) \mid\left(t_{1}, \ldots, t_{n}, 1\right) \in M_{l, m}\right\}$ and for $\left(t_{1}, \ldots, t_{n}\right) \in$ $Q_{l, m}, H_{\underline{d}}(t, 1, x)=(h(g f))_{\underline{d}}(t, x)$. To complete the proof we will check the 
well defining and the coherence conditions of the map $H_{\underline{d}}$. To check that the definition is well, suppose that $\left(t_{1}, \ldots, t_{n}, s\right) \in M_{l, m} \cap \bar{M}_{l-1, m}$, i.e., $t_{i}=\frac{2+s}{4}$. For these points $H_{\underline{d}} \frac{j}{d}$ is defined in two ways. If we compute the formula for $t=\left(t_{1}, \ldots, t_{n}, s\right) \in M_{l, m}$ and $t_{l}=\frac{2+s}{4}$, then we have:

$H_{\underline{d}}(t, s, x)$

$=h_{d_{0} \ldots d_{l-1}}\left(\frac{4 t_{1}-2-s}{2-s}, \ldots, \frac{4 t_{l-1}-2-s}{2-s}\right.$,

$\left.g_{h\left(d_{l} \ldots d_{m}\right)}\left(4 t_{l+1}-1-s, \ldots, 4 t_{m}-1-s, f_{g h\left(d_{m} \ldots d_{n}\right)}\left(\frac{4 t_{m+1}}{1+s}, \ldots, \frac{4 t_{n}}{1+s}, x\right)\right)\right)$.

The same expression is obtained if we compute the formula for $\left(t_{1}, \ldots, t_{n}, s\right) \in$ $M_{l-1, m}$ and $t_{l}=\frac{2+s}{4}$. Similarly, we can check that the definition is well for $\left(t_{1}, \ldots, t_{n}, s\right) \in M_{l, m} \cap M_{l, m-1}$, and the other cases can be deduced to one of these two cases. To check the coherence conditions of the homotopy $H_{\underline{d}}$, suppose that $\left(t_{1}, \ldots, t_{n}, s\right) \in M_{l-1, m}$ and $t_{n}=0$. Then $f_{g h\left(d_{m} \ldots d_{n}\right)}\left(\frac{\overline{4} t_{m+1}}{1+s}, \ldots, \frac{4 t_{n}}{1+s}, x\right)$, and it follows that for $t_{n}=0, H_{\underline{d}}=$ $H_{d_{0} \ldots d_{n-1}}\left(t_{1}, \ldots, t_{n-1}, x\right)$. The case when $t_{1}=0$ is treated similarly. If $t_{i}=t_{i+1}$, and $i<l$, then

$$
\begin{aligned}
& H_{\underline{d}}(t, s, x) \\
& =h_{d_{0} \ldots d_{i}}\left(\frac{4 t_{1}-2-s}{2-s}, \ldots, \frac{4 t_{i-1}-2-s}{2-s} \frac{4 t_{i+1}-2-s}{2-s}, \ldots, \frac{4 t_{l-1}-2-s}{2-s},\right. \\
& \left.g_{h\left(d_{i} \ldots d_{m}\right)}\left(4 t_{l+1}-1-s, \ldots, 4 t_{m}-1-s, f_{g h\left(d_{m} \ldots d_{n}\right)}\left(\frac{4 t_{m+1}}{1+s}, \ldots, \frac{4 t_{n}}{1+s}, x\right)\right)\right) \\
& =H_{d_{0} \ldots \hat{d}_{i} \ldots d_{n}}\left(t_{1}, \ldots, \hat{t}_{i}, \ldots, t_{n}, s, x\right) .
\end{aligned}
$$

The cases $l<i<m$ and $m<i<n$ are treated similarly.

THEOREM 5.2. If proximate coherent nets $f, f^{\prime}: X \rightarrow Y$ are homotopic, and coherent maps $g, g^{\prime}: Y \rightarrow Z$ are level homotopic, then the coherent maps $g f, g^{\prime} f^{\prime}: X \rightarrow Y$ are level homotopic.

Proof. Let $f, f^{\prime}: X \rightarrow Y$ be homotopic by a homotopy $F: I \times X \rightarrow Y$ given by a strictly increasing function $g: B \rightarrow A$. Then the proximate coherent nets $g f, g f^{\prime}: X \rightarrow Z$ are homotopic by the homotopy $g F: I \times X \rightarrow Z$ given by a strictly increasing function $f g: C \rightarrow A$. Let $g, g^{\prime}: Y \rightarrow Z$ be homotopic by a homotopy $G: I \times Y \rightarrow Z$ given by the strictly increasing function $g: C \rightarrow B$. Then the proximate coherent nets $g f^{\prime}, g^{\prime} f^{\prime}: Z \rightarrow Z$ are homotopic by the homotopy $G\left(1 \times f^{\prime}\right): I \times X \rightarrow Z$ given by strictly increasing function $f g: C \rightarrow A$. It follows that the proximate coherent nets $g f, g^{\prime} f^{\prime}: X \rightarrow Z$ are homotopic. 
THEOREM 5.3. The proximate coherent nets $f$ and $f 1_{X}$ are homotopic; $f$ and $1_{Y} f$ are homotopic.

PROOF. We will prove that $f$ and $1_{Y}$ are homotopic, and the other statement is treated in the similar way. First we define a partition of $\Delta^{n} \times I$ into subpolyhedra $L_{l}, l=0,1, \ldots, n$, by $L_{l}=\left\{\left(t_{1}, \ldots, t_{n}, s\right) \mid t_{l} \geq \frac{s}{2}+\frac{1}{2} \geq t_{l+1}\right\}$. We define a homotopy $F: I \times X \rightarrow Y$. This map will be given by the function $f: B \rightarrow A$ and by the maps $F_{\underline{b}}: \Delta^{n} \times I \times X_{f\left(b_{n}\right)} \rightarrow Y_{b_{0}}$ defined for $(t, s) \in L_{l}$ by $F_{\underline{b}}(t, s, x)=f_{b_{1} \ldots b_{n}}\left(\frac{2 t_{l}}{1+s}, \ldots, \frac{2 t_{n}}{1+s}, x\right)$. We mention that $K_{l} \times 0=$ $\left\{\left(t_{1} \ldots, t_{n}, 0\right) \mid\left(t_{1} \ldots, t_{n}, 0\right) \in L_{l}\right\}$ and then, for $t=\left(t_{1} \ldots, t_{n}\right) \in K_{l}$, we have $F_{\underline{b}}(t, 0, x)=\left(1_{Y} f\right)_{\underline{b}}(t, x)$. Also, $\left\{\left(t_{1}, \ldots, t_{n}, 1\right) \mid\left(t_{1}, \ldots, t_{n}\right) \in \Delta^{n}\right\}=L_{0}$ and $F_{\underline{b}}(t, 1, x)=f_{\underline{b}}(t, x)$. Category of strong shape is obtained. The objects are paracompact topological spaces, and the morphisms are the classes of the coherent proximate nets. For the isomorphic objects in this category we say they have the same strong shape.

\section{REFERENCES}

[1] B. Andonoviḱ, N. Shekutkovski, Subcategory of metric compacta in the strong shape category, Mat. Bilten 34 (2010), 13-25.

[2] K. Borsuk, Theory of shape, Polish Scientific Publishers, Warszawa, 1975.

[3] F.W. Cathey, J. Segal, Strong shape theory and resolutions, Topology Appl. 15 (1983), 119-130.

[4] D.A. Edwards, H.M. Hastings, Cech and Steenrod homotopy theories with applications to geometric topology. Lecture Notes in Mathematics 542, Springer, Berlin Heidelberg New York, 1976.

[5] J.E. Felt, E-continuity and shape, Proc. Amer. Math. Soc. 46 (1974), 426-430.

[6] Yu. Lisica and S. Mardešić, Coherent prohomotopy and strong shape of metric compacta, Glas. Mat. Ser. III 20(40) (1985), 159-186.

[7] S. Mardešić and J. Segal, Shape theory, North-Holland, Amsterdam, 1982.

[8] S. Mardešić, Strong shape and homology, Springer-Verlag, Berlin, 2000.

[9] J.B. Quigley, An exact sequence from the $n$-th to the $(n-1)$-st fundamental group, Fund. Math. 77 (1973), 195-210.

[10] J.M.R. Sanjurjo, A noncontinuous description of the shape category of compacta, Quart. J. Math. Oxford Ser. (2) 40 (1989), 351-359.

[11] N. Shekutkovski, Intrinsic definition of strong shape for compact metric spaces, Topology Proc. 39 (2012), 27-39.

[12] N. Shekutkovski, Shift and coherent shift in inverse systems, Topology Appl. 140 (2004), 111-130.

[13] N. Shekutkovski and B. Andonovik, Intrinsic definition of strong shape of strong paracompacta, Proceedings of IV Congress of the mathematicians of Republic of Macedonia, Struga, October 18-22, 2008, 2010, 287-299.

B. Andonovic

Faculty of Technology and Metallurgy

Ss Cyril and Methodius University

1000 Skopje

Macedonia

E-mail: beti@tmf.ukim.edu.mk 
N. Shekutkovski

Faculty of Mathematics and Natural Sciences

Ss Cyril and Methodius University

1000 Skopje

Macedonia

E-mail: nikita@pmf.ukim.mk

Received: 25.1.2016.

Revised: 1.11.2016. 